\title{
Consent - a new era begins
}

\author{
L. D'Cruz ${ }^{* 1}$ and H. Kaney ${ }^{2}$
}

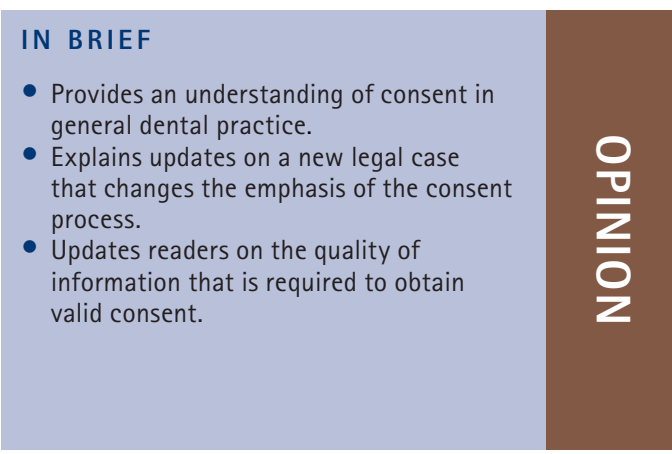

\begin{abstract}
The law in relation to consent has changed following the handing down of the Supreme Court judgement in Montgomery vs Lanarkshire in March this year. Legally, clinicians, including dentists, must now take reasonable care to ensure that patients are aware of any material risks involved in a proposed treatment and of reasonable alternatives. This case now brings the law in relation to the disclosure of risks when obtaining consent to treatment in line with the guidance issued by regulatory bodies such as the GMC and GDC.
\end{abstract}

\section{INTRODUCTION}

The law in relation to consent has changed following the handing down of the Supreme Court judgement in Montgomery vs Lanarkshire Health Board ${ }^{1}$ in March this year.

Legally, clinicians, including dentists, must now take reasonable care to ensure that patients are aware of any material risks involved in proposed treatment and of reasonable alternatives. This case now brings the law in relation to the disclosure of risks when obtaining consent to treatment in line with the guidance issued by regulatory bodies, that is, the GMC and GDC.

\section{THE FACTS OF THE CASE}

In 1999, Nadine Montgomery was pregnant with her first child. As a Type 1 insulin dependent diabetic, expectant mother, there was a risk of her carrying a large baby, as women with diabetes are likely to have babies that are larger than normal and there can be a particular concentration of weight on the babies' shoulders. This brings a risk of shoulder dystocia, a complication arising when the shoulders of the baby get stuck behind the pelvis during normal delivery, the risk being in the region of $9-10 \%$ in diabetic mothers.

Shoulder dystocia during delivery carries risks to the mother (in 11\% of cases there is post-partum haemorrhage and in $3.8 \%$ of

${ }^{1}$ GDP and Dento-legal Adviser, Dental Protection; ${ }^{2}$ Senior Dento-legal Adviser, Dental Protection

${ }^{*}$ Correspondence to: Dr Len D'Cruz

Email: lendcruz@btinternet.com

\section{Refereed Paper}

Accepted 10 June 2015

DOI: $10.1038 /$ sj.bdj.555

${ }^{\circledR}$ British Dental Journal 2015; 219: 57-59 cases there is fourth degree perineal tear) and to the baby. Seventy percent of cases of shoulder dystocia can be dealt with by the 'McRoberts' manoeuvre, but the manoeuvres used can cause shoulder and brachial plexus injury to the baby. The risk of brachial plexus injury is $0.2 \%$. In some cases, shoulder dystocia causes the umbilical cord to be trapped, causing hypoxia and cerebral palsy, the risk of this being less that $1 \%$.

During her ante-natal care, Mrs Montgomery raised concerns about standard delivery but her obstetrician did not warn her of the risks of shoulder dystocia, nor of any other risks that normal delivery carried. The obstetrician's rationale was that although there was a 9-10\% risk of shoulder dystocia (and it was accepted that this was a high risk), the risk of a grave problem resulting from shoulder dystocia was very low. The obstetrician explained during evidence that 'if you were to mention shoulder dystocia to every [diabetic] patient, if you were to mention to any mother who faces labour that there is a very small risk of the baby dying in labour, then everyone would ask for a caesarean section, and it's not in the maternal interests for women to have caesarean sections'. ${ }^{2}$ The Court ultimately did not like or accept this approach.

There was difficulty in delivering the baby as a result of shoulder dystocia and during the 12 minutes it took to free him he was starved of oxygen as the umbilical cord became occluded. The baby was born with cerebral palsy and suffered the loss of the use of his arm - a further complication of the brachial plexus injury sustained during the birth.

Mrs Montgomery raised an action alleging clinical negligence in the Court of Session in
Edinburgh and argued that had she known of the 10\% risk of shoulder dystocia, she would have asked for a caesarean section. The case was initially decided in favour of the defenders and an appeal to the Inner House of the Court of Session also failed. Mrs Montgomery therefore appealed the UK Supreme Court, based in London, which is the final court of appeal in the UK for civil cases, and for criminal cases from England, Wales and Northern Ireland.

\section{ANALYSIS OF THE JUDGEMENT AND THE PREVIOUS CASE LAW}

The Supreme Court, sitting with seven judges instead of the usual five, allowed the appeal and Mrs Montgomery was awarded £5.25 million in damages. In their analysis of the facts of the case, the Supreme Court considered the pre-existing case law in relation to disclosure of risks and the standard of care.

In Scotland, the legal test when alleging negligence stems from the case of Hunter $v s$ Hanley, ${ }^{3}$ which applies a three-stage test in order for breach of duty to be established. Firstly, it must be proved that there is a usual and normal practice. Secondly, it must be shown that the clinician did not adopt that practice. Thirdly, it must be proven that the course adopted by the clinician, is one that no clinician of ordinary skill would have taken if acting with ordinary care. The Hunter vs Hanley test was then adopted in England in 1957 in the Bolam case, ${ }^{4}$ in which it was established that a doctor is not guilty of negligence if acting in accordance with a practice accepted as proper by a responsible body of medical practitioners.

Various cases since then have applied the principles of Bolam and Hunter vs Hanley 
to claims concerning consent to treatment. In the case of Sidaway, ${ }^{5}$ the court applied the Bolam test to the issue of disclosure of risks when obtaining consent to treatment, although the court in Sidaway did indicate that there could be a situation in which the proposed treatment carried with it such grave risks that no prudent medical man would fail to warn of the risk. However, to all intents and purposes, the Bolam test was affirmed in relation to disclosure of risks. Sidaway was then followed in Scotland in the case of Moyes vs Lothian Health Board ${ }^{6}$ in which Lord Caplan said:

'In my view the Sidaway case in no way alters the pre-existing view of the law that the appropriate tests to apply in medical negligence cases are to be found in Hunter vs Hanley and Bolam.

However, the Australian case of Rogers vs Whitaker ${ }^{7}$ took a different approach in 1992 and, in that case, a clinician was found to be in breach of duty for failing to disclose the risk of total blindness to a patient who was already blind in one eye. This was perhaps a sign that the tide was turning, from the early 'doctor knows best' approach, through the responsible body of medical opinion test, to the specific patient approach which has now been stated in the Montgomery case. Bolitho, ${ }^{8}$ in 1998 , then emphasised that the medical profession does not solely dictate the standard of care of a doctor and that a judge is the final decision maker.

Until Montgomery, the test to be applied when disclosing risks was that of the prudent doctor/clinician and the tests laid out in Bolam and Hunter vs Hanley. That has now been overturned by the Montgomery case which overturned the decision made in Sidaway.

What the judges in Montgomery said was that the extent of information given to a patient about the risks of a proposed treatment is not to be determined by the clinician or what other clinicians in the same situation would do. Rather the test is what the particular patient sitting in front of the clinician wants to know. Patients must be told of material risks. The test of materiality is whether, in the circumstances of the particular case, a reasonable person in the patient's position would be likely to attach significance to the risk, or the clinician is or should reasonably be aware that the particular patient would be likely to attach significance to it.

\section{IMPLICATIONS FOR DENTISTRY}

What does this mean for dentists? In reality it changes professional practice very little and the Montgomery case simply brings the law into line with the ethical requirements imposed by the regulatory bodies, including the GDC. This has been the GDC position in Standards for the Dental Team since September 2013. However, the earlier GDC guidance and the GDC booklet on Principles of Patient Consent also required dentists to 'find out what your patients want to know as well as telling them what you think they need to know.' ${ }^{9}$ Dentists need to be fully familiar with the precise wording of Standards for the Dental Team and be aware of this legal case.

The requirement is that a dentist, in respect of consent, should tell the patient everything they want to know as well as everything the dentist thinks they might need to know. You might be forgiven for thinking that this has a mystic clairvoyant feel about it and you may not be far wrong. The intention is of course that the information asymmetry that exists in any professional relationship is levelled by the duty on the dentist to ensure that the patient has sufficient information to make an informed choice about what they want done to their bodies.

The obstetrician stated that had Mrs Montgomery asked her 'specifically about exact risks' she would have advised her of the risk of shoulder dystocia. A patient cannot be expected to know what questions to ask the clinician or to understand all of the implications of a procedure without expressly being informed about these or about alternatives. That is why they rely and trust the dentist in the first place. Equally important is the conclusion that the percentage risk of a situation occurring should not be the sole determinant of the disclosure of that risk to the patient. There are many percentages in clinical dentistry for example, in the case of loss of vitality after crown preparation or in relation to the success of root canal treatment. The significance of a given risk is likely to reflect a variety of factors besides its magnitude; for example, the nature of the risk, the effect which its occurrence would have upon the life of the patient, the importance to the patients of the benefits sought to be achieved by the treatment, the alternatives available and the risks involved in those alternatives. ${ }^{10}$

Thus simply quoting a percentage risk without putting it into the context of the patient's own views is insufficient.

The Montgomery judgement will perhaps require a little more time, effort and judicious questions being asked by the dentist to establish what is important to the patient and to ascertain what emphasis would need to be placed on any possible risk to them specifically. Depending on the setting of the care this may be more challenging when insufficient time is available to have these discussions. The prudent dentist should also record in detail the treatment options and risks discussed with the patient.

Where a patient does not wish to be informed of the risks and prefers not to discuss the matter at all then the clinician is not obliged to have these discussions. ${ }^{11}$

How we communicate those risks to patients or describe treatments is a significant part of the consent process. What emphasis should be placed on each of the risks and how determinative will it be in influencing a patient? There are risks for all dental procedures. Looking at root canal treatment of a heavily restored upper molar tooth $v s$ extraction will elicit many risks that final year dental students would be able to list in graphic detail. Indeed that is sometimes the very issue when obtaining consent. How much we tell patients about how the treatment is carried out, with a list of every possible option with their complications, sequelae and management of the complications can be influential and "too much in the way of information may only serve to confuse or alarm the patient'. The Montgomery case resolutely rejects this idea of paternalism and the notion that the clinician alone should decide what should be disclosed to patients.

\section{CONCLUSION}

The Montgomery case clarifies that clinicians must recognise a patient's legal and ethical right to autonomy and informed choice and sweeps away any notion that doctor (or dentist) knows best. Patients must be fully informed about the risks to them that any procedure carries and be permitted to decide whether to proceed at all or whether they wish to pursue alternative options or no treatment at all.

It is for the profession now to determine, clinical area by clinical area, what the risks that ought to be disclosed are and then it is for the individual clinician to decide what emphasis they need to place on those risks to the particular patient they have in their dental chair.

The moral of the story for dentists is that it is prudent to get to know your patients so that you can discuss with them risks that any patient would want to know, plus any risks that you would consider would be relevant to the particular patient concerned. In order to be able to satisfy the latter requirement, patients need to be asked if there is anything of particular relevance to them that they would want to know. The clinician's advice must be both 'fact sensitive and sensitive also to the characteristics of the patient. ${ }^{13}$ 
1. Montgomery (Appellant) $v$ Lanarkshire Health Board (Respondent) (Scotland) [2015] UKSC 104

2. Montgomery (Appellant) $\vee$ Lanarkshire Health Board (Respondent) (Scotland) [2015] UKSC 104 paragraph 13.

3. Hunter v Hanley [1955] SC 200.

4. Bolam v Friern Hospital Management Committee 19571 WLR 582.

5. Sidaway $\vee$ Board of Governors of the Bethlem Royal Hospital [1985] UKHL 1.
6. Moyes v Lothian Health Board [1990] SLT 444.

7. Rogers $\vee$ Whitaker [1992] HCA 58.

8. Bolitho $v$ City and Hackney Health Authority 1998 AC 232.

9. General Dental Council. Principles of patient consent.

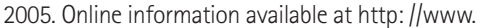
gdc-uk.org/Dentalprofessionals/Standards/Documents/ PatientConsent\%5B1\%5D.pdf (accessed June 2015).

10. Montgomery (Appellant) v Lanarkshire Health Board (Respondent) (Scotland) [2015] UKSC 104 paragraph 89.

11. Montgomery (Appellant) $\vee$ Lanarkshire Health Board (Respondent) (Scotland) [2015] UKSC 104 paragraph 85.

12. Montgomery (Appellant) $\vee$ Lanarkshire Health Board (Respondent) (Scotland) [2015] UKSC 104 paragraph 33.

13. Montgomery (Appellant) $\vee$ Lanarkshire Health Board (Respondent) (Scotland) [2015] UKSC 104 paragraph 89.

\section{Erratum}

\section{BDJ 2015; 218: 609-660 (Issue 11)}

The following digital object identifier (DOI) numbers associated with articles in issue 11 were incorrect in the original issue as published on the 12 June 2015. We apologise for any inconvenience caused by this error.

In Stephen Hancocks, Right handedness, BDJ 2015; 218: 609, the DOI was incorrectly stated as 10.1038/sj.bdj.2015.398. The correct number is 10.1038/sj.bdj.2015.477

In C. M. Murray \& N. P. Chandler, Magnification: Magnifying the point, BDJ 2015; 218: 610, the DOI was incorrectly stated as 10.1038/sj.bdj.2015.399. The correct number is $10.1038 /$ sj.bdj.2015.478

In P. Ramsay-Baggs, Good practice: The gloves are on, BDJ 2015; 218: 610, the DOI was incorrectly stated as 10.1038/sj.bdj.2015.400. The correct number is 10.1038/sj.bdj.2015.479 In H. Gayathri \& B. Madhan, Case report: Malingering and factitious disorders, BDJ 2015; 218: 610 , the DOI was incorrectly stated as $10.1038 / 5 j . b d j .2015 .401$. The correct number is 10.1038/sj.bdj.2015.480

In D. Howarth, Fluoride varnish: Coating over FV, BDJ 2015; 218: 610-611, the DOI was incorrectly stated as 10.1038/sj.bdj.2015.402. The correct number is 10.1038/sj.bdj.2015.481 In R. W. Mills, Safeguarding children, BDJ 2015; $218: 610$, the DOI was incorrectly stated as 10.1038/sj.bdj.2015.403. The correct number is 10.1038/sj.bdj.2015.482

In Letter (BDJ 2015; 215: 556-557), BDJ 2015; 218: 611, the DOI was incorrectly stated as 10.1038/sj.bdj.2015.404. The correct number is 10.1038/sj.bdj.2015.483

In C. D. Stephens, Orthodontics: Getting straight to orthodontic relapses, BDJ 2015; 218: 611 , the DOI was incorrectly stated as $10.1038 /$ sj.bdj.2015.405. The correct number is 10.1038/sj.bdj.2015.484

In Children's dental health - a mother's perspective, BDJ 2015; 218: 612-613, the DOI was incorrectly stated as 10.1038/sj.bdj.2015.406. The correct number is 10.1038/sj.bdj.2015.485 In Failure to include dentistry in health hurting patients and taxpayers, BDJ 2015; 218: 613, the DOI was incorrectly stated as 10.1038/sj.bdj.2015.407. The correct number is $10.1038 /$ sj.bdj.2015.486

In New CDO announced, BDJ 2015; 218: 614, the DOI was incorrectly stated as 10.1038 / sj.bdj.2015.408. The correct number is 10.1038/sj.bdj.2015.487

In Be clear on costs, dentists urged, BDJ 2015; $218: 614$, the DOI was incorrectly stated as 10.1038/sj.bdj.2015.409. The correct number is 10.1038/sj.bdj.2015.488

In A day in the life: 12 people. Eight days. One goal, BDJ 2015; $218: 614-615$, the DOI was incorrectly stated as 10.1038/sj.bdj.2015.410. The correct number is 10.1038/sj.bdj.2015.489 In BDA and BDIA announce new 'Strategic Partnership', BDJ 2015; 218: 616, the DOI was incorrectly stated as 10.1038/sj.bdj.2015.411. The correct number is 10.1038/sj.bdj.2015.490 In BDIA launches its 'Code of Practice for Dental CPD', BDJ 2015; 218: 616, the DOI was incorrectly stated as 10.1038/sj.bdj.2015.413. The correct number is 10.1038/sj.bdj.2015.491 In Bringing first smiles to children, BDJ 2015; $218: 616$, the DOI was incorrectly stated as 10.1038/sj.bdj.2015.412. The correct number is 10.1038/sj.bdj.2015.492

In Book review: Cone beam computed tomography in orthodontics: indications, insights and innovations, BDJ 2015; 218: 617, the DOI was incorrectly stated as 10.1038/ sj.bdj.2015.414. The correct number is 10.1038/sj.bdj.2015.493

In Book review: Mosby's orthodontic review, BDJ 2015; 218: 617, the DOI was incorrectly stated as 10.1038/sj.bdj.2015.415. The correct number is 10.1038/sj.bdj.2015.494

In Book review: Dentine hypersensitivity: Developing a person-centred approach to oral health, BDJ 2015; 218: 617, the DOI was incorrectly stated as 10.1038/sj.bdj.2015.416. The correct number is $10.1038 /$ sj.bdj.2015.495

In M. H. Thornhill et al., NICE and antibiotic prophylaxis to prevent endocarditis, BDJ 2015; 218: 619-621, the DOI was incorrectly stated as 10.1038/sj.bdj.2015.392. The correct number is $10.1038 /$ sj.bdj.2015.496

In J. C. Williams et al., Who wears the braces? A practical application of adolescent consent, BDJ 2015; 218: 623-627, the DOI was incorrectly stated as 10.1038/sj.bdj.2015.393.

The correct number is 10.1038/sj.bdj.2015.497

In J. M. Martin-Kerry et al., Practical considerations for conducting dental clinical trials in primary care, BDJ 2015; 218 : $629-634$, the DOI was incorrectly stated as 10.1038 / sj.bdj.2015.397. The correct number is 10.1038/sj.bdj.2015.498
In J. Radford, Commentary: Effect of beverages on color and translucency of new toothcolored restoratives, BDJ 2015; $218: 635$, the DOI was incorrectly stated as $10.1038 /$ sj.bdj.2015.417. The correct number is 10.1038/sj.bdj.2015.499

In J. Radford, Commentary: Choosing wisely in the UK: the Academy of Medical Royal Colleges' initiative to reduce the harms of too much medicine, BDJ 2015; 218: 635, the DOI was incorrectly stated as 10.1038/sj.bdj.2015.418. The correct number is 10.1038/sj.bdj.2015.500 In J. Radford, Commentary: Management of severe acute dental infections, BDJ 2015; 218: 635 , the DOI was incorrectly stated as $10.1038 /$ s..bdj.2015.419. The correct number is 10.1038/sj.bdj.2015.501

In J. Radford, Commentary: Infiltration and sealing versus fluoride treatment of occlusal caries lesions in primary molar teeth. $2-3$ years results, BDJ 2015; $218: 635$, the DOI was incorrectly stated as 10.1038/sj.bdj.2015.420. The correct number is 10.1038/sj.bdj.2015.502 In S. Wright \& $C$. Archer, An exploratory study to investigate if patients are able to aid the early diagnosis of peri-implant complications, BDJ 2015; 218: 637-640, the DOI was incorrectly stated as 10.1038/sj.bdj.2015.394. The correct number is 10.1038/sj.bdj.2015.503 In M. Ross \& S. Turner, Direct access in the UK: what do dentists really think?, BDJ 2015. 218: 641-647, the DOI was incorrectly stated as 10.1038/sj.bdj.2015.395. The correct number is 10.1038/sj.bdj.2015.504

In S. Kotecha et al., A multicentre audit of GDPs knowledge of orthodontic retention, BDJ 2015; 218: 649-653, the DOI was incorrectly stated as 10.1038/sj.bdj.2015.396. The correct number is 10.1038/sj.bdj.2015.505

In Supporting dental healthcare professionals, BDJ 2015; 218: 657, the DOI was incorrectly stated as 10.1038/sj.bdj.2015.421. The correct number is 10.1038/sj.bdj.2015.506

In All smiles, BDJ 2015; 218: 657, the DOI was incorrectly stated as 10.1038/sj.bdj.2015.422. The correct number is $10.1038 /$ sj.bdj.2015.507

In Results every time, BDJ $2015 ; 218: 657$, the DOI was incorrectly stated as 10.1038 / sj.bdj.2015.423. The correct number is 10.1038/sj.bdj.2015.508

In It's an easy life with efficient technology, BDJ 2015; 218 : 658 , the DOI was incorrectly stated as 10.1038/sj.bdj.2015.424. The correct number is 10.1038/sj.bdj.2015.509 In Get connected, BDJ 2015; 218: 658, the DOI was incorrectly stated as 10.1038 / sj.bdj.2015.425. The correct number is 10.1038/sj.bdj.2015.510

In A dental chair for you, BDJ 2015; 218 : 658 , the DOI was incorrectly stated as 10.1038 / sj.bdj.2015.427. The correct number is 10.1038/sj.bdj.2015.511

In The first purely ceramic-based restorative material, BDJ 2015; $218: 658$, the DOI was incorrectly stated as 10.1038/sj.bdj.2015.426. The correct number is 10.1038/sj.bdj.2015.512 In Extremely small and lightweight, BDJ 2015; $218: 659$, the DOI was incorrectly stated as 10.1038/sj.bdj.2015.428. The correct number is 10.1038/sj.bdj.2015.513

In Safer, easier, cheaper, BDJ 2015; 218: 659, the DOI was incorrectly stated as 10.1038/ sj.bdj.2015.429. The correct number is 10.1038/sj.bdj.2015.514

In Helpful convenience for orthodontic patients, BDJ 2015; 218: 659, the DOI was incorrectly stated as 10.1038/sj.bdj.2015.430. The correct number is 10.1038/sj.bdj.2015.515 In Dental care with extracts of ginger and curcuma, BDJ 2015; 218: 659, the DOI was incorrectly stated as 10.1038/sj.bdj.2015.431. The correct number is $10.1038 /$ sj.bdj.2015.516 In The next generation superbug beating technology has arrived, BDJ 2015; 218: 660, the DOI was incorrectly stated as $10.1038 /$ sj.bdj.2015.432. The correct number is $10.1038 /$ sj.bdj.2015.517

In Total patient comfort, BDJ 2015; 218: 660, the DOI was incorrectly stated as 10.1038 / sj.bdj.2015.435. The correct number is 10.1038/sj.bdj.2015.518

In Maximising your potential, BDJ 2015; $218: 660$, the DOI was incorrectly stated as 10.1038/sj.bdj.2015.433. The correct number is 10.1038/sj.bdj.2015.519

In Incorporating technology could be easier than you think, BDJ 2015; 218: 660, the DOI was incorrectly stated as $10.1038 /$ sj.bdj.2015.434. The correct number is 10.1038 / sj.bdj.2015.520 